\title{
Modified Cauchy Problem with Impulse Action for Parabolic Shilov Equations
}

\author{
Galina Unguryan (iD \\ Yu Fed'kovych Chernivtsi National University, 2 Kotsubins'ky Street, 58012 Chernivtsi, Ukraine \\ Correspondence should be addressed to Galina Unguryan; galuna_unguryan@ukr.net
}

Received 20 January 2021; Revised 5 April 2021; Accepted 15 April 2021; Published 28 April 2021

Academic Editor: Sergejs Solovjovs

Copyright (C) 2021 Galina Unguryan. This is an open access article distributed under the Creative Commons Attribution License, which permits unrestricted use, distribution, and reproduction in any medium, provided the original work is properly cited.

For parabolic Shilov equations with continuous coefficients, the problem of finding classical solutions that satisfy a modified initial condition with generalized data such as the Gelfand and Shilov distributions is considered. This condition arises in the approximate solution of parabolic problems inverse in time. It linearly combines the meaning of the solution at the initial and some intermediate points in time. The conditions for the correct solvability of this problem are clarified and the formula for its solution is found. Using the results obtained, the corresponding problems with impulse action were solved.

\section{Introduction}

In the medium $\mathbb{R}^{n}$, we consider a certain process whose evolution $u(t ; x)$ during the time $(0 ; T]$ is described by the partial differential equation:

$$
\begin{aligned}
\partial_{t} u(t ; x) & =P\left(t ; i \partial_{x}\right) u(t ; x), \\
(t ; x) \in \Pi_{(0 ; T]} & :=(0 ; T] \times \mathbb{R}^{n},
\end{aligned}
$$

in which

$$
P\left(t ; i \partial_{x}\right)=\sum_{|k| \leq p} a_{k}(t) i^{|k|} \partial_{x}^{k}
$$

differential expression of order $p>1$ with continuous coefficients $a_{k}(\cdot)$ on the set $[0 ; T]$. Here, $i$ is an imaginary unit, $\partial_{x}^{k}$ is a partial derivative of a variable $x$ of order $k$, and $\mathbb{R}^{n}$ is a real Euclidean space of dimension $n$ with a scalar product $(\cdot, \cdot)$ and norm $\|x\|:=(x, x)^{1 / 2}$.

We consider that equation (1) on the set $\Pi_{[0 ; T]}$ is a uniformly parabolic Shilov equation with the index of parabolicity $h, 0<h \leq p$, i.e., such that

$$
\exists \delta>0 \exists \delta_{0} \geq 0 \forall t \in[0 ; T] \forall \xi \in \mathbb{R}^{n}: \operatorname{Re} P(t ; \xi) \leq-\delta\|\xi\|^{h}+\delta_{0} .
$$

A simpler example (1) is the classical thermal conductivity equation

$$
\partial_{t} u(t ; x)=a^{2} \Delta u(t ; x), \quad(t ; x) \in \Pi_{(0 ; T]},
$$

with the Laplace operator $\Delta$, which describes the process of heat propagation or diffusion in the medium $\mathbb{R}^{n}$. This equation is also related to the random Wiener process. Its fundamental solution is the density distribution of the probability of motion of microscopic particles in a liquid or gas under the action of medium molecules.

If the initial state $f(x), x \in \mathbb{R}^{n}$, is known, this process at time $t=0$, i.e., the initial condition is set,

$$
u(t ; \cdot)_{\mid t=0}=f,
$$

then to find its evolution $u(t ; x)$ we get the Cauchy problem (1) and (5).

The study of the Cauchy problem (1) and (5) has been presented in many research works. In particular, authors in $[1,2]$ describe the classes of unity and correctness of this problem. The stabilization properties of the solutions of equation (1) under special $\Lambda$-conditions were studied in [3-5]. In [6-8], the alternative methods of the fundamental solution study are offered, which allow to avoid the notion of the equation kind (1) and difficulties associated with its 
location. The abstract theory of the Cauchy problem (1) and (5) in Banach spaces is developed in $[9,10]$. The works [11-16] are devoted to the construction of the theory of the Cauchy problem for equation (1) with variable coefficients. The results of these studies naturally complement and generalize the classical theory of the Cauchy problem for parabolic Petrovsky equations [17-19].

The Cauchy problem is a so-called direct problem. However, in many cases, there is a situation when it is necessary to study the evolution of the $u(t ; x)$ process described by equation (1), based only on the known information about its final (or intermediate) state in time:

$$
u(t ; \cdot)_{\mid t=T}=f,
$$

for example, astrophysical problems concerning the celestial bodies study and some problems of thermophysics, diffusion, demography, and mathematical biology (see [20-23]). Problem (1) and (6) is called an inverse problem. Usually, such a problem is incorrectly set by Hadamard [24].

Among the existing methods for solving the inverse problem of thermal conductivity, the method of quasireversibility should be noted [25]. However, this method is associated with an increase in the order of the original differential equation, which leads to significant difficulties in its numerical implementation. The Tikhonov regularization method for solving the equivalent integral equation [24] is an alternative to the quasireversibility method. In [26], developing Tikhonov's idea, a method for finding an approximate solution of the inverse thermal conductivity problem is proposed. It is based on the replacement of this problem by a corresponding problem with a nonlocal by time condition, in which a partial shift of the condition is carried out at $t=T$ at the initial moment $t=0$ :

$$
u(t ; \cdot)_{\mid t=0}+v u(t ; \cdot)_{\mid t=T}=f,
$$

where $v$ is a known parameter.

For parabolic Petrovsky equations, i.e., equation (1), in which $p=h=2 b$, problems with condition (7), and those with the conditions of a more general form were considered in [27-30]. Here various questions concerning the correctness of such problems and methods of solving them under certain conditions on the input data are considered. In this case, similar problems for parabolic Shilov equations with $p \neq h$ still remain in the state of expectation.

It should be noted that (1) and (6) is the problem of studying the evolution of a process in the past on the interval $(0 ; T)$ based on the data available about it $f(x), x \in \mathbb{R}^{n}$, at the moment of time $t_{*}=T$. However, if the information about the evolution of this process is also important for us in the near future, then we should already consider the problem for equation (1) with the condition

$$
u(t ; \cdot)_{\mid t=t_{*}}=f,
$$

for $t_{*} \leq T$. In this regard, the corresponding condition (7) takes the form

$$
u(t ; \cdot)_{\mid t=0}+v u(t ; \cdot)_{\mid t=t_{*}}=f .
$$

Most of the observed processes are influenced by impulses that are not taken into account by the differential equation of the corresponding mathematical model. Therefore, these effects should be reflected in the form of additional conditions to achieve the desired compliance of the model with the real process. Problems with impulse action for differential equations have been studied in many works, in [31-39] in particular. In [31, 32], the basics of the results of the theory of systems of differential equations with impulse action are presented. In [34], sufficient conditions are established for the controllability of a class of semilinear impulsive integrodifferential systems with nonlocal initial conditions in Banach spaces. The internal approximate controllability of the semilinear impulse deterministic thermal equation is set in [35]. In [36], this issue is already clarified for the semilinear impulse stochastic equation of thermal conductivity with delay. The Cauchy problem with impulse action for parabolic Petrovsky equations was studied in [37-39]. The abovementioned works and the references given in them refer to direct problems with impulse action, while inverse problems of this type have escaped the attention of researchers.

Let us return to the inverse problem (1) and (8). Suppose that we somehow managed to find out that at a certain point in time $t_{i}, t_{i} \neq t_{*}$, the process in question (1) was (or will be) subjected to a momentum effect of $\chi$ units. Then, model (1) and (8) must be supplemented by an additional impulse condition

$$
u\left(t_{i}^{+} ; \cdot\right)-u\left(t_{i}^{-} ; \cdot\right)=\chi
$$

where $u\left(t_{i}^{ \pm} ; \cdot\right):=\lim _{t \longrightarrow t_{i}}+0 u(t ; \cdot)$ and $\lim _{t \longrightarrow t_{i}}-0 u(t ; \cdot)$. In this case, if $t_{i}<t_{*}$, then problem (1), (8), and (10) is naturally called an inverse problem with an impulse precursor, in other words, an inverse problem with an impulse after-effect.

In this research, we study the modified Cauchy problem (1) and (9), which is generated by the inverse problem (1) and (8). By reducing to the corresponding Cauchy problem (1) and (5) in combination with the Fourier transform method, the correct solvability of this problem in a wide class of generalized initial data, such as the Gelfand and Shilov distributions, is established. At the same time, the explicit formulae of its classical solutions are found and the smoothness of these solutions by temporal and spatial variables is clarified. The results obtained are applied to solving the original problem (1) and (9) with the available time impulse (10), which can occur both before the time $t_{*}$ and after it.

The structure of the work is as follows. Section 2 provides the necessary information about the spaces of basic and generalized functions, which will serve as an environment for the study of the modified problem. The information about the correct solvability of the Cauchy problem for parabolic Shilov equations (1) is also presented here. In Section 3, the classical solutions of the modified Cauchy problem (1) and (9) with generalized initial data $f$ are found and their uniqueness is substantiated. Problem (1) and (9) with impulse after-effect and preeffect is solved in Sections 4 and 5, respectively. Section 6 presents the conclusions. 


\section{Preliminary Information}

Let $\mathbb{C}^{\infty}\left(\mathbb{R}^{n}\right)$ be a class of all functions infinitely differentiable on $\mathbb{R}^{n}$ and $S$ be the space of $L$. Schwartz elements from $\mathbb{C}^{\infty}\left(\mathbb{R}^{n}\right)$ rapidly fall to infinity, and $S^{\prime}$ is the corresponding space of Schwartz distributions [40]. The set of all $n$-dimensional multi-indices is denoted by $\mathbb{Z}_{+}^{n}$. And let $|l|=l_{1}+\cdots+l_{n}, z^{l}=z_{1}^{l_{1}}, \ldots, z_{n}^{l_{n}}$, if $z=\left(z_{1} ; \ldots ; z_{n}\right) \in \mathbb{R}^{n}$, $l=\left(l_{1} ; \ldots ; l_{n}\right) \in \mathbb{Z}_{+}^{n}$.

For $\alpha>0$ and $\beta>0$,

$$
\begin{aligned}
& S_{\alpha}=\left\{\varphi \in S\left|\exists A>0 \forall k \in \mathbb{Z}_{+}^{n} \exists c_{k}>0 \forall q \in \mathbb{Z}_{+}^{n} \forall x \in \mathbb{R}^{n}:\right| x^{q} \partial_{x}^{k} \varphi(x) \mid \leq c_{k} A^{|q|} q^{\alpha q}\right\} ; \\
& S^{\beta}=\left\{\varphi \in S\left|\exists B>0 \forall q \in \mathbb{Z}_{+}^{n} \exists c_{q}>0 \forall k \in \mathbb{Z}_{+}^{n} \forall x \in \mathbb{R}^{n}:\right| x^{q} \partial_{x}^{k} \varphi(x) \mid \leq c_{q} B^{|k|} k^{\beta k}\right\} ; \\
& S_{\alpha}^{\beta}=\left\{\varphi \in S\left|\exists A>0 \exists B>0 \exists c>0 \forall\{k, q\} \subset \mathbb{Z}_{+}^{n} \forall x \in \mathbb{R}^{n}:\right| x^{q} \partial_{x}^{k} \varphi(x) \mid \leq c A^{|q|} B^{|k|} q^{\alpha q} k^{\beta k}\right\} .
\end{aligned}
$$

The sets $S_{\alpha}, S^{\beta}$, and $S_{\alpha}^{\beta}$ with corresponding topologies [40] are countably normalized complete perfect spaces, which are called Gelfand and Shilov spaces of the type $S$.

The correct topological relationship is $S_{\alpha}^{\beta}=S_{\alpha} \cap S^{\beta}$ [41].

The space $S_{\alpha}^{\beta}$ is nontrivial for $\alpha+\beta \geq 1$ and consists only of those functions $\varphi \in \mathbb{C}^{\infty}\left(\mathbb{R}^{n}\right)$ that satisfy the inequality

$$
\left|\partial_{x}^{k} \varphi(x)\right| \leq c B^{|k|} k^{\beta k} e^{-\delta\|x\|^{1 / \alpha}}, \quad k \in \mathbb{Z}_{+}^{n}, x \in \mathbb{R}^{n},
$$

with positive constants $c, B$, and $\delta$, dependent on the function $\varphi$ only [40].
In spaces of the type $S$, continuous addition, multiplication, convolution, and the operator $F$ of the Fourier transform are defined, and the following topological equations are satisfied [40]: $F\left[S_{\alpha}\right]=S^{\alpha}, F\left[S^{\beta}\right]=S_{\beta}$, and $F\left[S_{\alpha}^{\beta}\right]=S_{\beta}^{\alpha}$.

We shall denote by $\Phi^{\prime}$ the space topologically conjugate to the space $\Phi \in\left\{S^{(1 / h)} ; S_{\beta}^{(1 / h)}, \beta \geq(p-1 / h)\right\}$, where $h>0$ and $p>1$.

For $\alpha_{1} \leq \alpha_{2}$ and $\beta_{1} \leq \beta_{2}$, the following continuous inclusions are satisfied [40]:

$$
S_{\alpha_{1}}^{\beta_{1}} \subset S_{\alpha_{2}}^{\beta_{2}} \subset\left\{\begin{array}{c}
S^{\beta_{1}} \subset S^{\beta_{2}} \\
S_{\alpha_{1}} \subset S_{\alpha_{2}}
\end{array}\right\} \subset S \subset S^{\prime} \subset\left\{S^{\beta_{2}^{\prime}} \subset S^{\beta_{1}^{\prime}} S_{\alpha_{2}}^{\prime} \subset S_{\alpha}^{\prime}\right\} \subset S_{\alpha_{2}}^{\beta_{2}^{\prime}} \subset S_{\alpha_{1}}^{\beta_{1}^{\prime}} .
$$

The Fourier transform of the generalized function $f \in \Phi^{\prime}$ and the convolution $f * g$ of the elements $\{f, g\} \subset \Phi^{\prime}$ are determined by the relations [40]:

$$
\langle F[f], F[\varphi]\rangle=(2 \pi)^{n}\langle f, \varphi\rangle ;\langle f * g, \varphi\rangle=\langle f, g * \varphi\rangle, \quad \varphi \in \Phi,
$$

where the angle brackets $\langle$,$\rangle indicate the effect of the$ generalized function on the basic one.

The convolution operation of the generalized function $f \in \Phi^{\prime}$ with the main function $\varphi \in \Phi$ is determined by the equality

$$
(f * \varphi)(\cdot)=\langle f(x), \varphi(x+\cdot)\rangle .
$$

The convolution $(f * \varphi)(\cdot)$ is a classic function from the class $\mathbb{C}^{\infty}\left(\mathbb{R}^{n}\right)[40]$.

It is obvious that the convolution operation $f * g$ in the space $\Phi^{\prime}$ will exist if the generalized function $g$ is a convoluter in the space $\Phi$, i.e., it is such that
(1) $(g * \varphi)(\cdot):=<g(x), \varphi(x+\cdot)>\in \Phi,(\forall \varphi \in \Phi)$

(2) The convolution operation $g$ with the elements $\varphi \in \Phi$ is continuous in the space $\Phi$

From here we come to the equality $F[f * g]=F[f] F[g]$, from which it becomes clear that the element $g \in \Phi^{\prime}$ is a convoluter in $\Phi$ only when its Fourier transform is $F[g]$-a multiplier in the corresponding space $F[\Phi]$.

The following criterion of the multiplier $[6,7]$ is correct: let $\theta_{\tau}^{t}(\cdot)=\exp \left\{\int_{\tau}^{t} P(\varsigma ; \cdot) \mathrm{d} \varsigma\right\}, \quad 0 \leq \tau<t \leq T$, then to be a multiplier in the space $F[\Phi]$, it is necessary and important for the function $\mu(\cdot) \in \mathbb{C}^{\infty}\left(\mathbb{R}^{n}\right)$ that for each fixed $t$, $0<t \ll 1$, the product $\left(\mu \theta_{0}^{t}\right)(\cdot)$ belongs to the space $F[\Phi]$.

In [6], the properties of the function $\theta_{\tau}^{t}(\cdot)$ are studied, in particular, the belonging of $\theta_{\tau}^{t}(\cdot)$ to the space $S_{(1 / h)}^{(p-1 / h)}$ for each fixed $t>\tau$, and the following estimates are obtained:

$$
\left|\partial_{\xi}^{k} \theta_{\tau}^{t}(\xi)\right| \leq c e^{\delta(t-\tau)} A^{|k|} k^{(p-1 / h) k}(t-\tau)^{n+\gamma(k)} e^{-\delta_{0}(t-\tau)\|\xi\|^{h}}, \quad k \in \mathbb{Z}_{+}^{n}, \xi \in \mathbb{R}^{n}, 0 \leq \tau<t \leq T,
$$


with positive constants $c, \delta, \quad \delta_{0}$, and $A$. Here, $\gamma(k)=(1-p)|k| / h$, if $0<t-\tau<1$ and $\gamma(k)=(1+h)|k| / h$ at $1 \leq t-\tau$.

We consider the Cauchy problem for equation (1) with the initial condition (5), in which $f$ is a functional from the space $\Phi^{\prime}$.

Definition 1. The solution of the Cauchy problem (1) and (5) on the set $\Pi_{(0 ; T]}$ is the function $u$, which on $\Pi_{(0 ; T]}$ satisfies equation (1) in the usual sense and the initial condition (5) in the sense of convergence in the space $\Phi^{\prime}$ :

$$
\langle u(t ; x), \varphi(x)\rangle \underset{t \rightarrow+0}{\longrightarrow}\langle f, \varphi(x)\rangle, \quad \forall \varphi \in \Phi .
$$

The fundamental solution of the Cauchy problem for equation (1) is a function

$$
G(t, \tau ; \cdot)=F^{-1}\left[\theta_{\tau}^{t}(\xi)\right](t, \tau ; \cdot), \quad 0 \leq \tau<t \leq T .
$$

Obviously, the solution $G(t, 0 ; \cdot) \in S_{(p-1 / h)}^{(1 / h)}$ for each fixed $t \in(0 ; T]$.

The next statement is correct.

Theorem 1 (see [6]). Let $f$ be a real functional from the space $\Phi^{\prime}$, then the corresponding Cauchy problem (1) and (5) on the set $\Pi_{(0 ; T]}$ is correctly solvable and its solution $u(t ; x)$ is differentiable with respect to the variable $t$ and infinitely differentiable with respect to the variable $x$, for which the following conditions are satisfied:

(1) $F\left[\partial_{t} u(t ; \cdot)\right]=\partial_{t} F[u(t ; \cdot)], t \in(0 ; T]$

(2) $u(t ; x)=f * G(t, 0 ; x),(t ; x) \in \Pi_{(0 ; T]}$

These results will help us to determine the correct solvability of the corresponding modified Cauchy problem (1) and (9), which will be considered in Section 3.

At the end of this section, we comment on conditions (1) and (2) of Theorem 1 for a better understanding of our further considerations. Since $G(t, 0 ; \cdot) \in \Phi, t \in(0 ; T]$, then according to equation (15), the solution of the Cauchy problem (1) and (5) is determined by the formula

$$
u(t ; \cdot)=\langle f(\xi), G(t, 0 ; \xi+\cdot)\rangle,
$$

with

$$
\begin{aligned}
\partial_{t} u(t ; x) & =\left\langle f(\xi), \partial_{t} G(t, 0 ; \xi+x)\right\rangle, \\
\partial_{x}^{k} u(t ; x) & =\left\langle f(\xi), \partial_{x}^{k} G(t, 0 ; \xi+x)\right\rangle,
\end{aligned}
$$

while the equality

$$
F\left[\partial_{t} u(t ; \cdot)\right]=\partial_{t} F[u(t ; \cdot)],
$$

is considered in the space $\Phi^{\prime}$.

\section{The Modified Cauchy Problem}

We arbitrarily fix the real-valued functional $f$ from the space $\Phi^{\prime}$, and for equation (1), we set the modified initial condition (9), which, considering the differentiability of the function $u$ at the point $t_{*}$, we regard as a weak convergence in $\Phi^{\prime}$ :

$$
u(t ; \cdot) \underset{t \rightarrow+0}{\stackrel{\Phi^{\prime}}{\longrightarrow}} f-v u\left(t_{*} ; \cdot\right)
$$

We shall solve the obtained problem (1) and (9) by the Fourier transform method.

To do this, we write the corresponding dual Fourier problem:

$$
\begin{aligned}
& \partial_{t} v(t ; \xi)=P(t ; \xi) v(t ; \xi), \quad(t ; \xi) \in \Pi_{(0 ; T]}, \\
& v(t ; \cdot) \underset{t \rightarrow+0}{\stackrel{F\left[\Phi^{\prime}\right]}{\longrightarrow}} \widetilde{f}-v v\left(t_{*} ; \cdot\right),
\end{aligned}
$$

where $v=F[u] \equiv \widetilde{u}$.

According to the classical Cauchy theorem, all solutions of equation (23) are described by the formula $v(t ; \cdot)=c(\cdot) \theta_{0}^{t}(\cdot)$ with an arbitrary function $c(\cdot)$. Hence, for $1+\nu \theta_{0}^{t_{*}}(\xi) \neq 0, \xi \in \mathbb{R}^{n}$, we arrive at the equivalence of the condition (24) to the following initial condition:

$$
v(t ; \cdot) \underset{t \rightarrow+0}{\stackrel{F\left[\Phi^{\prime}\right]}{\longrightarrow}} \frac{\tilde{f}}{1+\nu \theta_{0}^{t_{*}}(\cdot)} .
$$

Thus, for $1+\nu \theta_{0}^{t_{*}}(\xi) \neq 0, \quad \xi \in \mathbb{R}^{n}$, the dual Fourier problem (23) and (24) is equivalent to the Cauchy problem (23) and (25), and the only solution to these problems is the function

$$
v(t ; \xi)=\frac{\tilde{f}}{1+\nu \theta_{0}^{t_{*}}(\xi)} \theta_{0}^{t}(\xi), \quad(t ; \xi) \in \Pi_{(0 ; T]} .
$$

Then, taking into account the statement of Theorem 1, to prove the correct solvability of the original problem (1) and (9), it will be enough to substantiate the belonging of the functional $g=F^{-1}\left[\left(1+\nu \theta_{0}^{t_{*}}(\xi)\right)^{-1}\right] * f$ to the space $\Phi^{\prime}$ and its real-valuedness. For this, it would obviously be enough to show that the function $\mu_{\nu}(\cdot)=\left(1+\nu \theta_{0}^{t_{*}}(\cdot)\right)^{-1}$ is a multiplier in the space $F[\Phi]$.

Definition 2. We assume that for problems (1) and (9), the following condition holds:

$$
\inf _{\xi \in \mathbb{R}^{n}}\left|1+\nu \theta_{0}^{t_{*}}(\xi)\right| \neq 0
$$

In particular, condition (27) for problem (1) and (9) will be satisfied if $v \in \mathbb{R}^{n}$ is such that

$$
|\nu|<e^{-\delta_{0} t_{*}},
$$

where $\delta_{0}$ is the corresponding constant from the condition of parabolicity (3).

Indeed, directly from (3), we come to the estimate

$$
\left|\theta_{0}^{t_{*}}(\xi)\right| \leq e^{\delta_{0} t_{*}}, \quad \xi \in \mathbb{R}^{n} .
$$

Then, according to (28), we have

$\left|1+\nu \theta_{0}^{t_{*}}(\xi)\right| \geq|1-| \nu\left|\theta_{0}^{t_{*}}(\xi)\right| \geq 1-|\nu| e^{\delta_{0} t_{*}}>0, \quad \xi \in \mathbb{R}^{n}$. 
If the symbol $P(t ; \cdot)$ of equation (1) for each $t \in[0 ; T]$ acquires only valid values on $\mathbb{R}^{n}$, then condition (27) will already be satisfied for all $\nu>-e^{-\delta_{0} t_{*}}$.

Lemma 1. Suppose that condition (27) is satisfied for problem (1) and (9), then the corresponding function $\mu_{\nu}(\cdot)$ is a multiplier in the space $F[\Phi]$.
Proof. To simplify the calculations, we present a scheme of proof for the case $n=1$.

According to the well-known Faa di Bruno formula of the differentiation of a composite function

$$
\partial_{x}^{k} f(\varphi(x))=\sum_{r}^{k} \frac{k !}{q ! j !, \ldots, m !} \frac{\mathrm{d}^{r} f(\varphi)}{\mathrm{d} \varphi^{r}}\left(\frac{\mathrm{d} \varphi(x)}{1 ! \mathrm{d} x}\right)^{q}\left(\frac{\mathrm{d}^{2} \varphi(x)}{2 ! \mathrm{d} x}\right)^{j}, \ldots,\left(\frac{\mathrm{d}^{l} \varphi(x)}{l ! \mathrm{d} x}\right)^{m}, \quad k \in \mathbb{Z}_{+}, x \in \mathbb{R},
$$

where the sign of the sum extends to all integer nonnegative solutions of the equation $k=q+2 j+\cdots+1 \mathrm{~m}$, and $r=q+j+\cdots+m$, we obtain that

$$
\left|\partial_{\xi}^{k} \mu_{\nu}(\xi)\right| \leq \sum_{r}^{k} \frac{k !(r-1) !}{q ! j !, \ldots, m !}\left(\frac{|\nu|}{\inf _{\xi \in \mathbb{R}^{n}}\left|1+\nu \theta_{0}^{t_{*}}(\xi)\right|}\right)^{r}\left|\frac{\mathrm{d} \theta_{0}^{t_{*}}(\xi)}{1 ! \mathrm{d} x}\right|^{q}\left|\frac{\mathrm{d}^{2} \theta_{0}^{t_{*}}(\xi)}{2 ! \mathrm{d} x}\right|^{j}, \ldots,\left|\frac{\mathrm{d}^{l} \theta_{0}^{t_{*}}(\xi)}{l ! \mathrm{d} x}\right|^{m}, \quad k \in \mathbb{Z}_{+}, \xi \in \mathbb{R} .
$$

Hence, according to Stirling's formula

$$
n !=\sqrt{2 \pi n}\left(\frac{n}{e}\right)^{n}\left(1+O\left(\frac{1}{n}\right)\right)
$$

and the estimates (16),

$$
\frac{r !}{q ! j !, \ldots, m !} \leq 2^{k}
$$

we find out the existence of positive constants $c$ and $A$, such that for all $k \in \mathbb{Z}_{+}$and $\xi \in \mathbb{R}$, the following inequality is fulfilled:

$$
\left|\partial_{\xi}^{k} \mu_{\nu}(\xi)\right| \leq c A^{k} k^{(p-1 / h) k},
$$

which in combination with the estimate (16) ensures that the product $\left(\mu_{\nu} \theta_{0}^{t}\right)(\cdot)$ belongs to the space $S_{(1 / h)}^{(p-1 / h)}$ for each fixed $t \in(0 ; 1)$.

Therefore, $\mu_{v}(\cdot)$ is a multiplier in the space $S_{(1 / h)}^{(p-1 / h)}$.

Now that we have relation (13), we obtain the statement of the original lemma.

The lemma is proved.

We summarize the previous reflections in the form of the following statement.

Theorem 2. Suppose that condition (27) is satisfied and $f$ is a real-valued functional from the space $\Phi^{\prime}$, then the corresponding modified problem (1) and (9) on the set $\Pi_{(0 ; T]}$ is correctly solvable. Its solution $u$ is determined by the following formula:

$$
u(t ; x)=f * F^{-1}\left[\mu_{\nu}\right] * G(t, 0 ; x), \quad(t ; x) \in \Pi_{(0 ; T]} .
$$

In this case, $u(t ; x)$ is a classical function on $\Pi_{(0 ; T]}$, which is once differentiable with respect to the variable $t$ and infinitely differentiable with respect to the variable $x$, for which equality $(21)$ on the set $(0 ; T]$ is correct.

We now turn to consider the modified Cauchy problem (1) and (9) with a single impulse action that occurs at time $t_{i} \in(0 ; T)$. In this case, equation (1) will be considered on the set $\Pi_{(0 ; T] \backslash\left\{t_{i}\right\}}$ with an additional impulse condition (10), in which $\chi \in \mathbb{R} \backslash\{0\}$. We assume that the solution $u$ at the point $t_{i}$ is continuous on the left. Then we understand condition (10) in the following limiting limiting sense:

$$
u(t ; \cdot) \underset{t \rightarrow t_{i}+0}{\stackrel{\Phi^{\prime}}{\longrightarrow}} \chi+u\left(t_{i}^{-} ; \cdot\right) .
$$

Taking into account the specificity of condition (9), for $t_{*} \neq T$ there are two possible cases: $t_{*}<t_{i}$ (impulse after-effect) and $t_{i}<t_{*}$ (impulse preeffect).

We consider each of these cases separately.

\section{The Problem with Impulse After-Effect}

Let us consider here the situation when the impulse occurred after the "measurement" evolution $u(t ; \cdot)$ of the considered process, i.e., when $t_{*}<t_{i}$. In this case, on the set $\Pi_{(0 ; T] \backslash\left\{t_{i}\right\}}$, we have problem (1), (9), and (10) for $t_{*}<t_{i}$.

We shall solve this problem thinking as follows.

If we take into account the continuity on the left of the function $u(t ; \cdot)$ at the point $t_{i}$, then on the time interval $\left(0 ; t_{i}\right]$ the initial problem (1), (9), and (10) obviously reduces to problem (1) and (9) with $T=t_{i}$. Then, according to Theorem 2, under the appropriate conditions, the desired solution can be written as

$$
u(t ; x)=f * F^{-1}\left[\mu_{\nu}\right] * G(t, 0 ; x),(t ; x) \in \Pi_{\left(0 ; t_{i}\right]},
$$

with this solution being unique on the set $\Pi_{\left(0 ; t_{i}\right.}$. 
Further, the impulse condition (10) takes the form

$$
u(t ; \cdot)_{\mid t=t_{i}+0}=\varrho,
$$

where $\varrho=\chi+f * F^{-1}\left[\mu_{\nu}\right] * G\left(t_{i}, 0 ; \cdot\right)$-regular functionality from the space $\Phi^{\prime}$. Therefore, finding the solution $u$ of problem (1), (9), and (10) on the interval $\left(t_{i} ; T\right]$ is reduced to solving the Cauchy problem (1) and (39) on the set $\Pi_{\left(t_{i} ; T\right]}$. Using the statement of Theorem 1, we find that

$$
u(t ; x)=\varrho * G\left(t, t_{i} ; x\right), \quad(t ; x) \in \Pi_{\left(t_{i} ; T\right]},
$$

and this solution is also unique on $\Pi_{\left(t_{i} ; T\right]}$.

The use of the Heaviside function

$$
H(t)= \begin{cases}1, & 0<t, \\ 0, & t<0,\end{cases}
$$

enables to write the found solution on the set $\Pi_{(0 ; T] \backslash\left\{t_{i}\right\}}$ in the form

$$
u(t ; x)=\left[f * F^{-1}\left[\mu_{\nu}\right] * G(t, 0 ; x)\right] H\left(t_{i}-t\right)+\left[\left(\chi+f * F^{-1}\left[\mu_{\nu}\right] * G\left(t_{i}, 0 ; x\right)\right) * G\left(t, t_{i} ; x\right)\right] H\left(t-t_{i}\right)
$$

Hence, according to the convolution formula we get

$$
G(t, \tau ; \cdot)=G(t, \varsigma ; \cdot) * G(\varsigma, \tau ; \cdot), \quad 0 \leq \tau<\varsigma<t \leq T,
$$

$$
u(t ; x)=\left[f * F^{-1}\left[\mu_{\nu}\right] * G(t, 0 ; x)\right] H\left(t_{i}-t\right)+\left[\chi * G\left(t, t_{i} ; x\right)+f * F^{-1}\left[\mu_{\nu}\right] * G(t, 0 ; x)\right] H\left(t-t_{i}\right)
$$

Therefore, the following statement is correct.

Theorem 3. If condition (27) is satisfied and $f$ is a realvalued functional from the space $\Phi^{\prime}$, then for $t_{*}<t_{i}$ the corresponding problem (1), (9), and (10) on the set $\Pi_{(0 ; T] \backslash\left\{t_{i}\right\}}$ is correctly solved. Its solution $u(t ; x)$ is a classical function represented by formula (44). It is once differentiable with respect to the variable $t$ and infinitely differentiable with respect to the variable $x$, and equality (21) on the set $(0 ; T] \backslash\left\{t_{i}\right\}$ is correct.

\section{The Problem with Impulse Preeffect}

We consider now the case when the impulse occurs before the "measurement" evolution $u(t ; \cdot): t_{i}<t_{*}$. Then on the set $\Pi_{(0 ; T] \backslash\left\{t_{i}\right\}}$ we have problem (1), (9), and (10) for $t_{i}<t_{*}$. The next statement is correct.

Theorem 4. Let condition (27) be satisfied and $f$ be a realvalued functional from the space $\Phi^{\prime}$. Then the corresponding problem (1), (9), and (10) for $t_{i}<t_{*}$ on the set $\Pi_{(0 ; T] \backslash\left\{t_{i}\right\}}$ is correctly solvable and its solution $u(t ; x)$ is determined by the formula

$$
u(t ; x)=\left[\left(f-\nu \chi * G\left(t_{*}, t_{i} ; x\right)\right) * F^{-1}\left[\mu_{\nu}\right] * G(t, 0 ; x)\right] H\left(t_{i}-t\right)+\left[f * F^{-1}\left[\mu_{\nu}\right] * G(t, 0 ; x)+\chi * F^{-1}\left[\mu_{\nu}\right] * G\left(t, t_{i} ; x\right)\right] H\left(t-t_{i}\right)
$$

In this case, $u(t ; x)$ is a classical function on $\Pi_{(0 ; T] \backslash\left\{t_{i}\right\}}$, which is once differentiable with respect to the variable $t$ and infinitely differentiable with respect to the variable $x$, for which equation (21) is correct on the set $(0 ; T] \backslash\left\{t_{i}\right\}$.

Proof. The Fourier transform operator $F$ reduces problem (1), (9), and (10) to problem

$$
\begin{gathered}
\partial_{t} v(t ; \xi)=P(t ; \xi) v(t ; \xi), \quad(t ; \xi) \in \Pi_{(0 ; T] \backslash\left\{t_{i}\right\}} \\
v(t ; \cdot) \underset{t \rightarrow+0}{\stackrel{F\left[\Phi^{\prime}\right]}{\longrightarrow}} \widetilde{f}-v v\left(t_{*} ; \cdot\right),
\end{gathered}
$$

$$
v(t ; \cdot) \underset{t \rightarrow t_{i}+0}{\stackrel{F\left[\Phi^{\prime}\right]}{\longrightarrow}} \tilde{\chi}+v\left(t_{i}^{-} ; \cdot\right),
$$

where $v=\tilde{u}$.

According to the classical Cauchy theorem, all solutions of equation (46) on the set $[\tau ; T]$ are described by the formula $v(t ; \cdot)=c(\cdot) \theta_{\tau}^{t}(\cdot)$ with a random function $c(\cdot)$. Then the general solution of equation (46) on the set $[0 ; T] \backslash\left\{t_{i}\right\}$ is the set

$$
v(t ; \cdot)=c_{1}(\cdot) \theta_{0}^{t}(\cdot) H\left(t_{i}-t\right)+c_{2}(\cdot) \theta_{t_{i}}^{t}(\cdot) H\left(t-t_{i}\right),
$$

with random functions $c_{j}(\cdot)$.

From here and from conditions (47) and (48), we get the system 


$$
\left\{\begin{array}{l}
c_{1}(\cdot)+\nu c_{2}(\cdot) \theta_{t_{i}}^{t_{*}}(\cdot)=\tilde{f}, \\
c_{2}(\cdot)-c_{1}(\cdot) \theta_{0}^{t_{i}}(\cdot)=\tilde{\chi},
\end{array}\right.
$$

to find the quantities $c_{j}(\cdot) \in F\left[\Phi^{\prime}\right]$ for which the function $v$ from equality (49) is the solution to problem (46)-(48).

Provided that

$$
1+\nu \theta_{0}^{t_{*}}(\cdot) \neq 0
$$

system (50) has a single solution

$$
\begin{aligned}
& c_{1}(\cdot)=\frac{\tilde{f}-\nu \tilde{\chi} \theta_{t_{i}}^{t_{*}}(\cdot)}{1+\nu \theta_{0}^{t_{*}}(\cdot)}, \\
& c_{2}(\cdot)=\frac{\tilde{f} \theta_{0}^{t_{i}}(\cdot)+\tilde{\chi}}{1+\nu \theta_{0}^{t_{*}}(\cdot)} .
\end{aligned}
$$

Therefore, the function

$$
v(t ; \xi)=\frac{\tilde{f}-\nu \tilde{\chi} \theta_{t_{i}}^{t_{*}}(\xi)}{1+\nu \theta_{0}^{t_{*}}(\xi)} \theta_{0}^{t}(\xi) H\left(t_{i}-t\right)+\frac{\tilde{f} \theta_{0}^{t_{i}}(\xi)+\tilde{\chi}}{1+\nu \theta_{0}^{t_{*}}(\xi)} \theta_{t_{i}}^{t}(\xi) H\left(t-t_{i}\right), \quad(t ; \xi) \in \Pi_{(0 ; T] \backslash\left\{t_{i}\right\}},
$$

is the only solution of problem (46)-(48).

Let condition (27) be satisfied. Then acting on equality (53) by the operator $F^{-1}$, we obtain the solution of problem (1), (9), and (10)

$$
u(t ; \cdot)=F^{-1}\left[\mu_{\nu}(\xi)\left\{\left(\tilde{f}-\nu \tilde{\chi} \theta_{t_{i}}^{t_{*}}(\xi)\right) \theta_{0}^{t}(\xi) H\left(t_{i}-t\right)+\left(\tilde{f} \theta_{0}^{t_{i}}(\xi)+\tilde{\chi}\right) \theta_{t_{i}}^{t}(\xi) H\left(t-t_{i}\right)\right\}\right](t ; \cdot),
$$

which is also the only one. This solution allows images

$$
\begin{aligned}
u(t ; x)=[ & \left.\left(f-\nu \chi * G\left(t_{*}, t_{i} ; x\right)\right) * F^{-1}\left[\mu_{\nu}(\xi)\right] * G(t, 0 ; x)\right] H\left(t_{i}-t\right) \\
& +\left[\left(f * G\left(t_{i}, 0 ; x\right)+\chi\right) * F^{-1}\left[\mu_{\nu}(\xi)\right] * G\left(t, t_{i} ; x\right)\right] H\left(t-t_{i}\right) . \\
& f * g=F^{-1}[F[f] F[g]] .
\end{aligned}
$$

This is easy to see, if we take into account equation (18), the properties of the function $\theta_{\tau}^{t}(\cdot)$, statement of Lemma 1 , and the known formula

Further, if $t \in\left(0 ; t_{i}\right]$, then

$$
u(t ; x)=\left(f-\nu \chi * G\left(t_{*}, t_{i} ; x\right)\right) * F^{-1}\left[\mu_{\nu}(\xi)\right] * G(t, 0 ; x), \quad x \in \mathbb{R}^{n}
$$

and, if $t \in\left(t_{i} ; T\right]$, then

$$
u(t ; x)=\left(f * G\left(t_{i}, 0 ; x\right)+\chi\right) * F^{-1}\left[\mu_{\nu}(\xi)\right] * G\left(t, t_{i} ; x\right), \quad x \in \mathbb{R}^{n} .
$$

However, each of equations (57) and (58) determines the solution of the Cauchy problem (1) and (5) with the corresponding initial function on the corresponding time interval. Therefore, according to Theorem 1 , the found function $u$ is a classical solution of equation (1) on the set $\Pi_{(0 ; T] \backslash\left\{t_{i}\right\}}$, besides this $u(t ; \cdot) \in \mathbb{C}^{\infty}\left(\mathbb{R}^{n}\right),(0 ; T] \backslash\left\{t_{i}\right\}$, and the equality is satisfied

$$
F\left[\partial_{t} u(t ; \cdot)\right]=\partial_{t} F[u(t ; \cdot)], \quad t \in(0 ; T] \backslash\left\{t_{i}\right\} .
$$

Now applying the convolution formula (43) to equation (55), we come to the image of the solution of problem (1), (9), and (10) in the form (45).

The theorem is proved.

In conclusion, we demonstrate the obtained results on the example of the classical equation of thermal conductivity (4). The fundamental solution of the Cauchy problem for (4) is a function 


$$
G(t, \tau ; \cdot)=(2 a \sqrt{\pi(t-\tau)})^{-n} e^{-\left(\|\cdot\|^{2} / 4 a^{2}(t-\tau)\right)}, \quad 0 \leq \tau<t \leq T, \forall T>0,
$$

with

$$
\theta_{\tau}^{t}(\cdot)=e^{-a^{2}(t-\tau)\|\cdot\|^{2}}, \quad 0 \leq \tau<t \leq T .
$$

Since $P(t ; \xi)=-a^{2}\|\xi\|^{2} \in \mathbb{R}, \xi \in \mathbb{R}^{n}$, we choose $\nu>-1$. This choice holds condition (27) for the corresponding task (4) and (9).

Equation (4) is a Shilov parabolic one, for which $p=h=2$. Therefore, its fundamental solution is $G(t, 0 ; \cdot) \in S_{1 / 2}^{1 / 2}, t>0$.

Further, in the space $S_{1 / 2}^{1 / 2}$, we have

$$
F\left[F^{-1}\left[\mu_{\nu}\right] * G(t, 0 ; \cdot)\right]=\mu_{\nu} F[G(t, 0 ; \cdot)]=\frac{e^{-a^{2} t\|\cdot\|^{2}}}{1+\nu e^{-a^{2} t_{*}\|\cdot\|^{2}}} \equiv \phi, \quad t>0 .
$$

The functional $\phi$ is a regular generalized function with $S_{1 / 2}^{1 / 2^{\prime}}$ generated by the element $\phi(\cdot) \in S_{1 / 2}^{1 / 2}$. Then, $F^{-1}\left[\mu_{\nu}\right] G(t, 0 ; \cdot) \in S_{1 / 2}^{1 / 2}$, in this case, the following equality is satisfied

$$
F^{-1}\left[\mu_{\nu}\right] * G(t, 0 ; \cdot)=F^{-1}\left[\frac{e^{-a^{2} t\|\xi\|^{2}}}{1+v e^{-a^{2} t_{*}\|\xi\|^{2}}}\right](t ; \cdot), \quad t>0
$$

If we now choose a really significant generalized function $f$, the $\delta$-Dirac function from the space $S_{1 / 2}^{1 / 2}$, for the initial one, is then

$$
f * F^{-1}\left[\mu_{\nu}\right] * G(t, 0 ; \cdot)=F^{-1}\left[\frac{e^{-a^{2} t\|\xi\|^{2}}}{1+\nu e^{-a^{2} t_{*}\|\xi\|^{2}}}\right](t ; \cdot), \quad t>0 .
$$

Hence, according to formula (36), we obtain the following classical solution of the corresponding modified Cauchy problem (4) and (9):

$$
u(t ; x)=F^{-1}\left[\frac{e^{-a^{2} t\|\xi\|^{2}}}{1+v e^{-a^{2} t_{*}\|\xi\|^{2}}}\right](t ; x), \quad(t ; x) \in \Pi_{(0 ; T]} .
$$

The solution $u(t ; \cdot)$ for each $t>0$ is an element of the space $S_{1 / 2}^{1 / 2}$.

For $\chi$ from $\mathbb{R}$, we have

$$
\chi * G\left(t, t_{i} ; x\right)=\left\langle\chi, G\left(t, t_{i} ; \xi+x\right)\right\rangle=\chi \int_{\mathbb{R}^{n}} G\left(t, t_{i} ; \xi+x\right) \mathrm{d} \xi=\chi .
$$

Considering this, directly from formula (44), we arrive at the following solution of the modified Cauchy problem (4), (9), and (10) with impulse after-effect on the set $\Pi_{(0 ;+\infty) \backslash\left\{t_{i}\right\}}$ :

$$
u(t ; x)=H\left(t_{i}-t\right) F^{-1}\left[\frac{e^{-a^{2} t\|\xi\|^{2}}}{1+v e^{-a^{2} t_{*}\|\xi\|^{2}}}\right](t ; x)+H\left(t-t_{i}\right)\left[\chi+F^{-1}\left[\frac{e^{-a^{2} t\|\xi\|^{2}}}{1+v e^{-a^{2} t_{*}\|\xi\|^{2}}}\right](t ; x)\right]
$$

Obviously, $\quad u(t ; \cdot) \in S_{1 / 2}^{1 / 2}, \quad t \in\left(0 ; t_{i}\right), \quad$ and $u(t ; \cdot) \in \mathbb{C}^{\infty}\left(\mathbb{R}^{n}\right), t>t_{i}$.

In a similar way from formula (45), we obtain the solution of the modified Cauchy problem (4), (9), and (10) with impulse preeffect on the set $\Pi_{(0 ;+\infty) \backslash\left\{t_{i}\right\}}$ :

$$
\begin{aligned}
u(t ; x)= & {\left[F^{-1}\left[\frac{e^{-a^{2} t\|\xi\|^{2}}}{1+v e^{-a^{2} t_{*}\|\xi\|^{2}}}\right](t ; x)-\frac{v \chi}{1+v}\right] H\left(t_{i}-t\right) } \\
& +\left[F^{-1}\left[\frac{e^{-a^{2} t\|\xi\|^{2}}}{1+v e^{-a^{2} t_{*}\|\xi\|^{2}}}\right](t ; x)+\frac{\chi}{1+v}\right] H\left(t-t_{i}\right) .
\end{aligned}
$$

This solution is an element of the class $\mathbb{C}^{\infty}\left(\mathbb{R}^{n}\right)$ for each $t \in(0 ;+\infty) \backslash\left\{t_{i}\right\}$.

\section{Conclusions}

This research deals with a modified Cauchy problem for parabolic Shilov equations with variable coefficients, which arises at approximately solving the time-inverse parabolic problem. The correct solvability of this problem in a wide class of generalized initial data such as the Gelfant and Shilov distributions is determined. At that, the method of reducing the modified problem to the classical Cauchy problem is applied. This method allows obtaining important results about the solvability of the new problem in the form of corresponding consequences from the known statements about the Cauchy problem; this significantly simplifies the research process. Also, the correct solvability of this problem with the available single impulse is found. At the same time, the cases with impulse after-effect and preeffect in relation to the moment of "measurement" of evolution of the considered process are separately considered. For parabolic Shilov equations and in the case of the problem with impulse action, for parabolic Petrovsky equations as well, the results obtained here are new.

The formulae of classical solutions with generalized boundary values of these problems found here can be applied for virtual visualization of these processes using computer technology. They are also appropriate for 
numerical analysis using modern application packages. On the other hand, these results are important for further studies of parabolic equations with nonlocal and impulse conditions of a more general structure.

\section{Data Availability}

The data used in the research to support the findings of this study are purely bibliographic and from scientific publications, which are included in the article with their respective citations.

\section{Conflicts of Interest}

The author declares that he has no conflicts of interest.

\section{References}

[1] G. E. Shilov, "On conditions of correctness of Cauchy's problem for systems of partial differential equations with constant coefficients," Uspekhi Matematicheskikh Nauk, vol. 10, no. 4, pp. 89-100, 1955, in Russian.

[2] I. Gel'fand and G. Shilov, "Generalized functions," Theory of Differential Equations, Academic Press, Boston, MA, USA, 1967.

[3] S. D. Eidelman, S. D. Ivasishen, and F. O. Porper, "The Liouville theorems for systems parabolic by Shilov," Central European Journal of Mathematics, vol. 169, no. 6, p. 179, 1961 in Russian.

[4] V. V. Gorodetskii, "The Cauchy problem for Shilov parabolic equations in classes of generalized periodic functions," Central European Journal of Mathematics, vol. 10, no. 5, pp. 82-84, 1988, in Russian.

[5] V. V. Gorodetskii, "Some theorems on stabilization of solutions of the Cauchy problem for Shilov parabolic systems in classes of generalized functions," Ukrainian Mathematical Journal, vol. 40, no. 1, pp. 43-48, 1988, in Russian.

[6] V. A. Litovchenko, "The Cauchy problem for Shilov parabolic equations," Siberian Mathematical Journal, vol. 45, no. 4, pp. 669-679, 2004.

[7] V. A. Litovchenko, "Cauchy problem for -parabolic equations with time-dependent coefficients," Mathematical Notes, vol. 77, no. 3-4, pp. 364-379, 2005.

[8] V. A. Litovchenko, "One method for the investigation of fundamental solution of the Cauchy problem for parabolic systems," Ukrainian Mathematical Journal, vol. 70, pp. 922934, 2018.

[9] Q. Zheng, "Matrices of operators and regularized cosine functions," Journal of Mathematical Analysis and Applications, vol. 315, no. 1, pp. 68-75, 2006.

[10] M. Kostic, "Shilov Parabolic Systems," Bulletin, vol. 37, pp. 19-39, 2012.

[11] Y. I. Zhitomirskii, "Cauchy problem for some types of systems of linear equations, which are parabolic by G.E. Shilov, with partial derivatives with continuous coefficients," Izvestiya Rossiiskoi Akademii Nauk, vol. 23, pp. 925-932, 1959, in Russian.

[12] V. A. Litovchenko and I. M. Dovzhitska, "The fundamental matrix of solutions of the Cauchy problem for a class of parabolic systems of the Shilov type with variable coefficients," Journal of Mathematical Sciences, vol. 175, no. 4, pp. 450-476, 2011.
[13] V. A. Litovchenko and I. M. Dovzhytska, "Cauchy problem for a class of parabolic systems of Shilov type with variable coefficients," Central European Journal of Mathematics, vol. 10, no. 3, pp. 1084-1102, 2012.

[14] V. A. Litovchenko and I. M. Dovzhytska, "Stabilization of solutions to Shilov-type parabolic systems with nonnegative genus," Siber Mathematics Journal, vol. 55, no. 2, pp. 276-283, 2014.

[15] V. A. Litovchenko and G. M. Unguryan, "Conjugate Cauchy problem for parabolic Shilov type systems with nonnegative genus," Differential Equations, vol. 54, no. 3, pp. 335-351, 2018.

[16] V. Litovchenko and G. Unguryan, "Some properties of Green's functions of Shilov-type parabolic systems," Miskolc Mathematical Notes, vol. 20, no. 1, pp. 365-379, 2019.

[17] I. G. Petrovskii, "On the Cauchy problem for systems of equations with partial derivatives in a domain of nonanalytic functions," Bulletin, vol. 1, no. 7, pp. 1-72, 1938, in Russian.

[18] S. D. Eidelman, Parabolic Systems, North-Holland, Amsterdam, Netherland, 1969.

[19] A. Friedman, Partial Differential Equations of Parabolic Type, Prentice-Hall, Englewood Cliffs, NJ, USA, 1964.

[20] J. R. Cannon and J. van der Hoek, "Diffusion subject to the specification of mass," Journal of Mathematical Analysis and Applications, vol. 115, no. 2, pp. 517-529, 1986.

[21] A. M. Nakhushev, Equations of Mathematical Biology, Springer, Berlin, Germany, in Russian, 1995.

[22] I. A. Belavin, S. P. Kapitsa, and S. P. Kurdyumov, "Mathematical model of global demographic processes taking into account spatial distribution," Computational Mathematics and Mathematical Physics, vol. 38, no. 6, pp. 885-902, 1998, in Russian.

[23] S. M. Alekseeva and N. I. Yurchuk, "The quasi-inversion method for the initial condition control problem for the heat equation with integral boundary condition," Differential Equation, vol. 34, no. 4, pp. 495-502, 1998, in Russian.

[24] A. N. Tikhonov and V. Y. Arsenin, Methods for Solving Incorrect Problems, Springer, Berlin, Germany, 1979, in Russian.

[25] R. Lattes and J.-L. Lions, Methode de quasi-reversibilite et applications, Dunod, Paris, France, 1967.

[26] P. N. Vabishchevich, "Nonlocal parabolic problems and the inverse problem of thermal conductivity," Differential Equation, vol. 17, no. 7, pp. 1193-1199, 1981, in Russian.

[27] N. I. Ivanchov, "Boundary value problems for a parabolic equation with Integral Conditions," Differential equation, vol. 40, pp. 591-609, 2004.

[28] J. Chabrowski, "On the non-local problems with a functional for parabolic equation," Funckcialaj Ekvacioj, vol. 27, pp. 101-123, 1984.

[29] V. V. Shelukhin, "Time-nonlocal problem for the equations of the dynamics of a barotropic ocean," Siber Mathematics Journal, vol. 36, no. 3, pp. 701-724, 1995, in Russian.

[30] V. V. Gorodetskiy, R. S. Kolisnyk, and O. V. Martynyuk, "On a nonlocal problem for partial diferential equations of parabolic type," Bukovinian Mathematics Journal, vol. 8, no. 2, pp. 24-39, 2020.

[31] V. Lakshmikantham and D. D. Bainov, Simeonov Theory of Impulsive Differential Equations, World Scientific, Singapore, 1989.

[32] A. M. Samoilenko and N. A. Perestyuk, Impulsive Differential Equations, World Scientific, Singapore, 1995.

[33] V. E. Slyusarchuk, "General theorems on the existence and uniqueness of solutions of differential equations with 
impulsive action," Ukrainian Mathematical Journal, vol. 52, no. 7, pp. 954-964, 2000, in Russian.

[34] B. Radhakrishnan and K. Balachandran, "Controllability results for semilinear impulsive integrodifferential evolution systems with nonlocal conditions," Journal of Control Theory and Applications, vol. 10, no. 1, pp. 28-34, 2012.

[35] H. Leiva and N. Merentes, "Approximate controllability of the impulsive semilinear heat equation," Journal of Mathematics and Applications, vol. 38, pp. 85-104, 2015.

[36] H. Leiva, M. Narvaez, and Z. Sivoli, "Controllability of impulsive semilinear stochastic heat equation with delay," International Journal of Differential Equations, vol. 2020, Article ID 2515160, 10 pages, 2020.

[37] M. I. Matiichuk and V. M. Luchko, "Cauchy problem for parabolic systems with pulse action," Ukrainian Mathematical Journal, vol. 58, no. 11, pp. 1525-1535, 2006.

[38] L. P. Luo, Y. Q. Wang, and Z. G. Gong, "New criteria for oscillation of vector parabolic equations with the influence of impulse and delay," Acta Scientiarum Naturalium Universitatis Sunyatseni, vol. 51, no. 2, pp. 45-48, 2012.

[39] I. D. Pukal'skii and B. O. Yashan, "The Cauchy problem for parabolic equations with degeneration," Advances in Mathematical Physics, vol. 2020, Article ID 1245143, 7 pages, 2020.

[40] I. M. Gel'fand and G. E. Shilov, "Spaces of basic and generalized functions,"Elsevier, Amsterdam, Netherlands, 1958, in Russian.

[41] A. I. Kashpirovsky, "About coincidence of spaces $S_{\alpha} \cap S^{\beta}$ and $S_{\alpha}^{\beta}$ Functional analysis and its application," vol. 14, no. 2, pp. 60-72, 1980, in Russian. 